\title{
Traditional Chinese Medicine View on Fighting against COVID-19
}

\author{
Tong Zheng* \\ Department of Biomedical sciences, China
}

*Corresponding author: Tong Zheng, Department of Biomedical sciences, China.

Received Date: June 08, 2020

Published Date: June 19, 2020

\begin{abstract}
The sudden outbreak of COVID-19 is leading the health systems around the world not to acquire the characteristics of this novel coronavirus with the lack of pathological data on COVID-19. The successful treatments of influenza A and B and Enterovirus (EV) suggest that the generating sequence of Zang-Fu organs deserves attention since edema found in the patient's lungs is viewed as the pattern of deficient Spleen qi.
\end{abstract}

Keywords: COVID-19; Generating sequence; Spleen qi deficiency.

\section{Introduction}

Compared to SARS, a novel coronavirus originated from Beijing, China in 2002, COVID-19 that broke out in Wuhan, China, in December 2019 is surely challenging to the health system around the world. The infection is affecting 213 countries, areas or territories up to April, 2020 and deserves attention.

Traditional Chinese medicine (TCM) and acupuncture composed of the major philosophical and abstract concepts like YinYang, the Five Elements, Qi, Blood, Pattern, and Wei-Qi-Yin-Blood sequence are not based on modern science but have been viewed as the major healthcare tool in China for more than two thousand years. These unique and abstract concepts distinguish absolutely TCM and acupuncture based on the inductive reasoning and the observation of the nature from the Western medicine derived from the deductive reasoning. Both TCM and acupuncture are not only accepted in the Chinese communities, but also in Asian countries like Hong Kong, Macau, Japan, Taiwan, Singapore, and Korea. At present, acupuncture is also recommended by the WHO for pain management with its efficacy and has been recognized and gained the popularity in the West, though it's still viewed and classified as primarily the complementary or alternative medicine [1].

From the perspective of TCM or acupuncture, a disease is the presentation of the imbalance between Yin referring to material and Yang representing internal Zang-Fu organs' functions. The imbalance serves as the foundation of Pattern that varies among people with unique constitution [2]. In other words, a disease is treated according to an individual's pattern, which is the key to the successful TCM, acupuncture, and acupressure treatments. A Pattern, however, must be identified strictly with the Yin-Yang theory for prescriptions $[3,4]$.

The Five Elements theory refers to the five elements - wood, fire, earth, metal, and water as shown in Figure 1 and is used to describe interactions and relationships between things, while each element corresponds to an organ. This theory not only presents the fundamental elements of everything in the universe where interactions occur [5], but also can be applied to the balance of organs with the notion that both TCM and acupuncture treat a body 
as a whole system and a miniature of the universe. This theory suggests TCM and acupuncture are seeking the similarities of the disease instead of the differences the Western medicine focuses on (Figure 1) (Table 1)

Table 1: Elements corresponding to organs.

\begin{tabular}{|c|c|c|c|c|c|}
\hline Elements & Metal & Wood & Water & Fire & Earth \\
\hline Organs (Yin) & Lung & Liver & Kidney & Heart & Spleen \\
\hline Organs (Yang) & Large Intestine & Gall Bladder & Urinary Bladder & Small Intestine & Stomach \\
\hline
\end{tabular}

Figure: Five Elements.

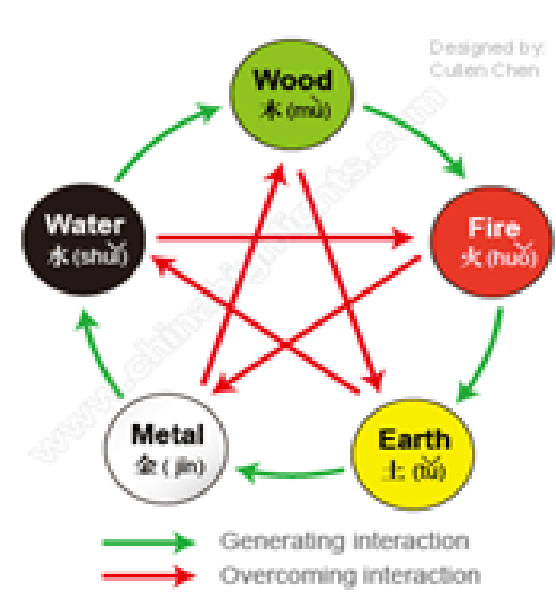

This article aims to discuss whether or not the generating sequence may be an option in fighting against the COVID-19. There are 6 confirmed deaths of 397 cases in Taiwan as of April 18, 2020 and what needs discussion and attention is that three deaths out of the six death cases have one thing in common-diabetes, which is accurately the presentation of Spleen qi deficiency.

\section{Critical concept}

The past experiences treating influenza $A$ and $B$ and Enterovirus (EV) show that the Five Elements theory highlights the importance of the generating sequence of Zang-Fu organs to maintain the balance and coordination of the organs.

With accurate diagnosis based on the generating sequence theory derived from Yin-Yang and the Five Elements rather than the diseases classified by the Western medicine, the results that those patients who were treated with TCM herbs or acupuncture recovered very soon only with 1-2 treatments, even overnight in some cases, indicate the organ generating sequence that Earth/ Spleen generates Metal/Lung of the Five Elements is logical and Table 2: Functions of Spleen and Lung. effective [6].

Spleen, foundation of postnatal existence, is the central organ to produce Qi and extract food Qi (Gu Qi) from food and liquids one takes into the body. Food Qi is the basis for the formation of Qi and Blood circulating in the body for the balance of Yin-Yang. On the other hand, Lung dominates the overall Qi facilitating the flow of energy throughout our body to exchange and regulate Qi in the body. In fighting against COVID-19, the functions of Spleen and Lung shown in Table 2 need attention [7] (Table 2).

Both symptomatic and asymptomatic transmissions of COVID-19 have been confirmed by the WHO [8]. Common symptoms of COVID-19 include fever, cough, and shortness of breath and some other symptoms like fatigue, abdominal pain, sore throat, loss of smell, muscle pain, and diarrhea can be seen in some cases [9]. Based on the TCM and acupuncture principles for classifying symptoms, these symptoms are classified in Table 3 in accordance with the functions of TCM Zan-organs (Table 3).

\begin{tabular}{|c|c|}
\hline Zang-organs & Functions \\
\hline \multirow{4}{*}{ Spleen } & Coverns transformation and transportation of Gu Qi and fluids \\
\cline { 2 - 2 } & Controls the Blood \\
\cline { 2 - 3 } & Controls the muscles and the four limbs \\
\cline { 2 - 3 } & Cons into the mouth and manifests in the lips \\
\cline { 2 - 3 } & Controls the raising of Qi, the "raising of the pure" \\
\cline { 2 - 3 } & The Spleen houses thought \\
\hline
\end{tabular}




\begin{tabular}{|c|c|}
\hline \multirow{4}{*}{ Lung } & Rule Qi and Respiration \\
\cline { 2 - 3 } & Control the Channels and Blood Vessels (with the Heart) \\
\cline { 2 - 3 } & Control dispersing and descending \\
\cline { 2 - 3 } & Regulate and move the Water Passages \\
\cline { 2 - 3 } & Control skin, sweat glands and body hair (the "Exterior") \\
\cline { 2 - 3 } & Open into the nose \\
\cline { 2 - 3 } & House the Po (corporeal soul) \\
\cline { 2 - 3 } & Govern the voice \\
\hline
\end{tabular}

Table 3: Classified symptoms corresponding to organs.

\begin{tabular}{|c|c|}
\hline Organs & Symptoms \\
\hline Spleen & fatigue, abdominal pain, muscle pain, diarrhea \\
\hline Lung & fever, cough, shortness of breath, sore throat, loss of smell \\
\hline
\end{tabular}

In the TCM and acupuncture theories, the constitution of humans is the key to the outcomes. The three essential and vital treasures Jing (essence), Qi, and Shen are the foundations of a human body. Jing (essence) bearing the high similarity to genes is viewed as Ying and as material. The damages to Jing may cause serious damages to physiological and psychological developments. Qi is always the vital energy consistently circulating through the body and classified as Yang for the body to fight against external evil Qi (pathogens) to maintain a person's health. In addition, Yang refers to transporting Blood to nourish the all the systems of the body. These three treasures do not coordinate with one other when an external evil Qi invades the body, resulting in the imbalance between Yin and Yang [10].

The TCM term for diabetes is Xiao-ke, which appears in the earliest TCM classic Huang Di Nei Jing (The Yellow Emperor's Inner Classic), means "wasting and thirsting."

Xiao-ke, which literally means in Chinese internal heat that consumes fluids to bring on wasting and thirsting, is attributed to three main factors, such as Yin deficiency in constitution, improper diet, and emotional disturbances. Three types of Xiao-ke are generally divided in accordance with the San Jiao (Triple Burner) system: upper, middle, and lower, while each has characteristic symptoms. Excessive thirst can be seen in the upper Jaio, the middle by is characterized by excessive hunger, and excessive urination in the lower. Pathologically these types are closely associated with Yin deficiency and in some cases Qi deficiency, particularly Spleen qi, in the Zang-organs of Lung, Stomach, and Kidney respectively [11]. Xiao-ke occurs once these three Zang-organs cannot coordinate with one another; for example, Spleen in qi deficiency fails to generate Lung.

Spleen generates Lung in the generating sequence of the Five Elements, which suggests how to prevent Lung from damage lies in healthy Spleen. Spleen is paired with Stomach and functions to transport and transform food and fluid in the body. Spleen qi deficiency develops with poor lifestyle habits like Emotional strain, exposure to a damp environment or damp weather, the improper diet for a long time, a genetic predisposition to Spleen imbalances, or using steroids and antibiotics to damage Spleen and finally leads to internal Dampness. In addition to the phenomenon of Damp resulted from external weather exposure, Spleen malfunctions to manage fluids correctly in the body when qi is deficient. The prolonged qi deficiency gradually makes Phlegm and edema produced with Dampness in the body when Dampness is not properly cleared. Both swelling and heaviness are the typical physical signs of Dampness [12,13,14].

\section{Practical suggestions}

In the eyes of TCM and acupuncture, COVID-19 is classified as the external evil Qi that can invade the human body to damage Spleen and the three treasures to cause serious health issues.

Pathologic examinations of two suspected COVID-19 patients who underwent lung lobectomies for adenocarcinoma revealed that apart from the tumors, their lungs exhibited edema, proteinaceous exudate, and etc. [15]. In the meantime, the condition of COVID-19 toes which involves purple coloring and inflammation of the toes is also believed to the possible sign of COVID-19 [16]. These cases suggest that "edema" is the key to treatment with TCM medications and acupuncture since it is accurately related to Spleen qi deficiency, based on the functions of Spleen that Spleen governs the four limbs and Lung controls skin.

As discussed above, Lung located in the Upper Jiao is generated by Spleen. The color of the toe that is controlled by Lung changes when Spleen qi is deficient to generate Lung. In other words, Lung is damaged when Spleen qi is deficient. This concept highlights that the consideration in treatment should be given to Spleen to maintain the regular and normal generating sequence.

Different from differentiating the viruses of SARS and MERS from COVID-19, the TCM and acupuncture practitioners should follow the Yin-Yang theory for the accurate Patter identification to treat a patient for the successful outcomes. It is reported that the exclusive and newly-developed protocol composed of Yuan-Source and Luo-Connecting acuoints was conducted to the patient at stage 
IV with urothelial carcinoma and the levels of cancer pain on the Wong-Baker FACES Pain Scale was reduced from 8 to 3 with four consecutive acupuncture treatments. This case indicates TCM and acupuncture should deserve attention in the public health system with the notion that different diseases can be treated with the same approach as long as the identical Pattern occurs [2].

The Spleen functions in this context are very different from those of the Western medicine, in which Spleen is viewed as a concrete organ for digestion. In terms of fighting against diseases, most of current evidence-based research unfortunately ignores the key to the successful outcomes-Pattern [5]. However, the cases of treating influenza A and B and Enterovirus (EV) show the organ generating sequence of the Five Elements theory surely deserves attention and consideration when fighting against COVID-19 since respiratory symptoms occurs among them.

It also deserves attention how to integrate TCM with the Western medicine. In general, Pattern identification of the TCM and acupuncture theories is a little bit difficult and may hinder patients, learners, and those who are interested in TCM and acupuncture from learning and understanding to get the whole picture of the TCM and acupuncture. In other words, the current evidencebased research and the trend of the integration of the East and the West medicines seem to suggest that there requires linking TCM/ acupuncture with the Western medicine to begin a conversation.

\section{Conclusion}

Pattern identification is absolutely the key to the successful TCM and acupuncture treatments. There is the lack of pathologic data on the novel COVID-19 at present, but the alternative approach may give to this notion of TCM and acupuncture that different diseases can be treated with the same approach, which is seldom considered in the evidence-based research. In truth, the efficacy of TCM, acupuncture, and acupressure cannot be examined accurately as long as most current researches still only focus on the characteristics of a disease or a virus. It is the right time to consider Pattern in search for the feasible solutions to fighting against COVID-19 event with the integration of TCM and acupuncture.

\section{Acknowledgement}

Not applicable.

\section{Conflicts of Interest}

No conflicts of interest.

\section{References}

1. Hong TZ (2019) A Close Look at the Application of the Yin-Yang-Based Acupoint Pairs. Advancements Bioequivalent.

2. Hong TZ (2018) Challenges in Learning and Understanding Traditional Chinese Medicine and Acupuncture. Open Access Journal of Complementary \& Alternative Medicine.

3. TZ Hong (2019) Clinical Challenges in Using Chinese Medicinal Herbs. Archives in Biomedical Engineering \& Biotechnology 2(3).

4. TZ. Hong (2017) Acupressure or Acupuncture at Sanyinjiao (SP6) for Primary Dysmenorrhea. Journal of Network Medicine and Target Therapies 1(1).

5. China Highlights. China's Five Elements Philosophy - Wood, Fire, Earth, Metal, and Water. China Highlights.

6. TZ. Hong (2017) Exploring a New Extra Point for Sub-acute Cough: A Case Report. Journal of Complementary Medicine \& Alternative Healthcare.3(4)

7. Sacred Lotus Chinese Medicine. Internal organs. Sacred Lotus Chinese Medicine.

8. WHO Coronavirus disease 2019 (COVID-19) Situation Report-73.

9. Centers for Disease Control and Prevention Symptoms. 2019 Novel Coronavirus. Centers for Disease Control and Prevention. US.

10. TZ Hong (2018) Understanding the TCM Role of Liver in the Treatment of Cough. LOJ Pharmacology \& Clinical Research 1(2)

11. MB Covington (2011) Traditional Chinese Medicine in the Treatment of Diabetes. Diabetes Spectrum 14(3): 154-159.

12. TZ Hong (2019) Managing Cancer Pain with the Exclusive and Newly Developed Reinforcing Yang-Nourishing Yin Acupoint Protocol. Canadian Journal of Biomedical Research and Technology 1(1).

13. Ageless Herbs Spleen Qi Deficiency.

14. TZ Hong (2018) Cautions for Learning Traditional Chinese Medicine and Acupuncture. Advancements in Bioequivalence \& Bioavailability 2(2).

15. Tian Sufang Pulmonary Pathology of Early-Phase 2019 Novel Coronavirus (COVID-19) Pneumonia in Two Patients with Lung Cancer. Journal of Thoracic Oncology.

16. Scripps National, Doctors say 'COVID toes' may be symptom of coronavirus, especially in youth. 